\title{
Caractérisation de matériaux céramiques soumis à rayonnements laser UV : applications au traitement de surface
}

\author{
Ch. Prat, G. Nicolas-Costa et M. Autric \\ Institut de Mécanique des Fluides, Equipe Interaction Laser-Matière, Parc Scientifique et \\ Technologique de Luminy, 13009 Marseille, France
}

\begin{abstract}
Résumé : Du fait de leur mode de fabrication, les matériaux céramiques offrent souvent un aspect granuleux ainsi qu'une rugosité et une porosité non négligeables. L'usinage mécanique en est, de plus, tendu délicat de par leur dureté. La présente étude vise donc à modifier et à caractériser l'état de surface de différents matériaux céramiques afin d'en améliorer les propriétés tribologiques. Des expériences ont été réalisées sur alumine $\left(\mathrm{Al}_{2} \mathrm{O}_{3}\right)$, nitrure d'aluminium (AlN), et carbure de silicium (SiC) au moyen de lasers à excimères afin d'étudier l'influence de divers paramètres tels que la longueur d'onde et la densité d'énergie sur la rugosité et la porosité des surfaces irradiées.
\end{abstract}

\section{CONDITIONS EXPERIMENTALES.}

Les échantillons sont irradiés au moyen de lasers impulsionnels susceptibles de délivrer des rayonnements de 193, 248 et $308 \mathrm{~nm}$ avec des durées d'impulsions de l'ordre de 25 ns. L'énergie est focalisée sur la surface à traiter de façon à atteindre des densités d'énergie de 0 à $8 \mathrm{~J} / \mathrm{cm}^{2}$.

Ces surfaces sont ensuite examinées par microscopie électronique à balayage (MEB) et au moyen d'un profilomètre mécanique. La rugosité en terme de Ra (moyenne arithmétique des valeurs absolues des écarts du profil à l'intérieur de la longueur de base) est calculée à partir de mesures effectuées sur une longueur d'évaluation minimale de 1,5 $\mathrm{mm}$ pour une longueur de base fixée à $0,25 \mathrm{~mm}$. Le rayon de la pointe de diamant est de 2 $\mu \mathrm{m}$.

Par visualisation et traitement d'images, l'évolution de la porosité (ce terme recouvrant indifféremment les pores et les interstices entre les grains constituant l'échantillon) a pu être quantifiée.

Des mesures d'énergie ąbsorbée sont également effectuées au moyen d'un thermocouple chromel-constantan.

\section{RESULTATS - DISCUSSION.}

\subsection{Seuils de transformation des matériaux.}

Les observations au MEB permettent d'estimer le seuil de fusion des matériaux ainsi que celui de disparition de la structure granulaire. Quant au seuil d'ablation, il est considéré atteint lorsqu'un cratère est détecté par le profilomètre après 40 ou 100 tirs. Les valeurs de ces seuils sont données dans le tableau 1 pour différentes longueurs d'onde. 
Seuil fusion

\begin{tabular}{|c|c|c|}
\hline 003 & 248 & $-1,3$ \\
\hline & 308 & 1,5 \\
\hline ic & $248 \mathrm{n}$ & 0 \\
\hline & $193 \mathrm{n}$ & $0,5-0,8$ \\
\hline $\begin{array}{l}\text { AIN - } \\
\text { AIN - }\end{array}$ & $\begin{array}{l}248 \mathrm{~nm} \\
193 \mathrm{~nm}\end{array}$ & $\begin{array}{l}<1 \\
\approx 0,5\end{array}$ \\
\hline
\end{tabular}

Seuil ablation

2

Tableau 1 : Valeurs des seuils de fusion, d'ablation et de disparitions des grains (en $\mathrm{J} / \mathrm{cm}^{2}$ )

Des mesures d'énergie absorbée effectuées à des densités d'énergie inférieures et supérieures au seuil estimé de fusion de l'alumine, révèlent également que l'évolution de l'absorptivité consécutive au changement d'état du matériau, apparait vers $1 \mathrm{~J} / \mathrm{cm}^{2}$.

\subsection{Porosité.}

La porosité totale minimale sur l'Al2 $\mathrm{O}_{3}$ est obtenue pour $2 \mathrm{~J} / \mathrm{cm}^{2}$ (à $248 \mathrm{~nm}$ ). Les interstices d'une largeur initiale de $1 \mu \mathrm{m}$, ne sont plus visibles mais, du fait de la rapidité du refroidissement de la zone irradiée, des microfissures apparaissent ainsi que des pores que l'on attribuer à l'existence de bulles dans le matériau en fusion. Cependant, l'état de surface parait être meilleur une fois la structure granulaire effacée à savoir pour des densités d'énergie incidentes de l'ordre de $3 \mathrm{~J} / \mathrm{cm}^{2}$ (fig.1).

\subsection{Rugosité.}

La rugosité initiale des échantillons étudiés est de $0,55,0,35$ et $0,15 \mu \mathrm{m}$ respectivement pour l'Al2 $\mathrm{O}_{3}$, l'AlN $^{2}$ et le $\mathrm{SiC}$. Ces valeurs ont tendance à diminuer (fig.2) dans la plage de densité d'énergie utilisée, excepté pour le $\mathrm{SiC}$ dont la surface se couvre de bourrelets dus à la solidification de matériau fondu. Cette morphologie rappelle celle d'un métal traité dans les mêmes conditions. De plus, la couleur métallique (argentée) acquise par l'échantillon traduit un enrichissement superficiel en $\mathrm{Si}$ au niveau de la surface irradiée 1 .

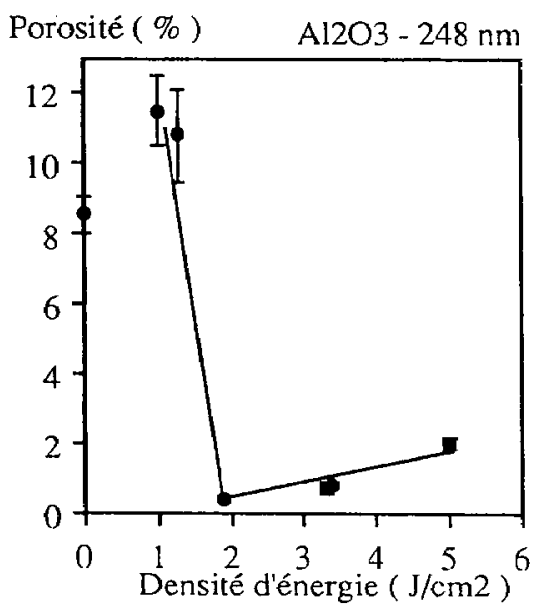

Fig. 1 : Evolution de la porosité de $1^{\prime} \mathrm{Al}_{2} \mathrm{O}_{3}$ en fonction de la densité d'énergie.

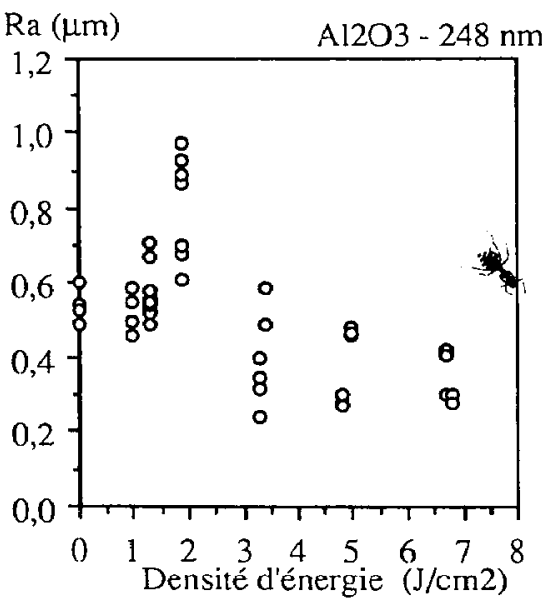

Fig.2 : Evolution de la rugosité de l'Al2 $\mathrm{O}_{3}$ en fonction de la densité d'énergie.

\section{Remerciements.}

Les auteurs tiennent à remercier Monsieur Serge Nitsche pour ses conseils lors de l'utilisation du microscope électronique à balayage. 